\section{Von der Produkt-}

\section{zur Bedürfnisorientierung}

\begin{abstract}
Den Unternehmen kommt eine Schlüsselrolle bei der umwelt- und sozialverträglichen Gestaltung unserer Gesellschaft zu. Soweit besteht Einigkeit. Es fragt sich aber, was dies für Unternehmen bedeutet, die nur dann überleben können, wenn ihre Kunden mit den Produkten zufrieden sind. Welche Anforderungen werden an Unternehmen gestellt, und wie kann öko-effiziente Produktgestaltung aussehen?
\end{abstract}

D

Von Kai Hockerts und Michael G. Moeller Wirtschaftskrise und des wachsenden, internationalen Wettbewerbsdrucks vor allem damit beschäftigt, ihre Geschäftsprozesse zu rationalisieren, um einerseits Kosten zu senken und andererseits die Zufriedenheit der Kunden mit den Leistungen des Unternehmens zu steigern. Zukunftsfähigkeit wird hier mit Wettbewerbsfähigkeit gleichgesetzt. Sie erfordert Produkte, die die Erwartungen der Kundschaft hinsichtlich Preis und Leistung erfuillen. Moderne Managementkonzepte wie Business Reengineering und Total Quality Management machen daher das dauerhafte Erkennen und Befriedigen der Kundenbedürfnisse zur Richtschnur für alle Unternehmensaktivitäten.

Ausgangspunkt der Umweltdiskussion ist dagegen der Schutz der natürlichen Umwelt: Hierfür wird u.a. gefordert, den Rohstoff- und Energieverbrauch der Industrieländer um 80 bis 90 Prozent zu senken. Eine Effizienzrevolution soll durch technologische Verbesserungen die Produktivität der natürlichen Ressourcen vervielfachen und die Umweltverträglichkeit von Produkten verbessern.

Beide Ansätze rücken stets die Rationalisierung des Mitteleinsatzes in den Vordergrund: hier der effiziente Einsatz der Produktionsfaktoren Kapital und Arbeit, dort die Produktivität der natürlichen Ressourcen. Für eine zukunftsfähige Gestaltung von Leistungen reichen sie aber nicht aus. Die Rationalität der Mittel kann die potentielle Irrationalität der gewählten Ziele des unternehmerischen Handelns nicht verhindern. Unter dem Stichwort Suffizienzrevolution wird daher gefordert, neue Leitbilder für den individuellen und gesellschaftlichen Wohlstand zu entwickeln.
Was aber bedeutet dies aus der Sicht eines Unternehmens, das sich Gedanken über die Gestaltung seiner Produkte macht?

\section{Anforderungen an die Unternehmen}

Macht sich ein Unternehmen an die Entwicklung neuer Produkte, so geht es zunächst darum, sinnvolle Ziele und Bewertungskriterien für Produkte festzulegen. Eine umweltverträgliche Produktgestaltung erfordert Ziele für den Verbrauch natürlicher Ressourcen und die Entstehung von Umweltbelastungen entlang des gesamten Produktlebensweges. Ein Produkt kann seinen Zweck aber nur dann erfïllen, wenn es die tatsächlịchen Bedürfnisse des Kunden befriedigt. Gerade hier liegt trotz Qualitätsmanagement und Marketing noch immer ein wesentlicher Schwachpunkt der Produktpolitik von Unternehmen: Allzu häufig geht man dort der Neigung nach, die bestehenden Produkte durch marginale Veränderungen an neue Nachfragetrends anzupassen. Häufig wird auch einfach ein bestimmter Bedarf als Ausgangspunkt der Leistungspolitik definiert, ohne zu fragen, welche menschlichen Bedürfnisse dahinter stehen. Dadurch bleibt unberïcksichtigt, daß ein bestimmter Bedarf immer auch Folge bestimmter technischer, gesellschaftlicher, politischer oder wirtschaftlicher Rahmenbedingungen ist, die selbst wiederum der Befriedigung menschlicher Bedürnnisse dienen sollen.

Die Festlegung produktbezogener Ziele erzwingt Werturteile, die eine Abwägung zwischen alternativen, widersprüchlichen Bedürfnissen erfordern. Letzlich müßte eine solche Feststellung im Diskurs aller Anspruchsgruppen des Unternehmens erfolgen, wofür es bislang kaum konkrete Verfahrensvorschläge gibt. Durch eine effiziente Gestaltung der materiellen und immateriellen Produktmerkmale gilt es dann in einem zweiten Schritt, eine möglichst öko-effiziente Befriedigung des Kundennutzens zu erreichen. Dabei steht allzu häufig allein die technische Umsetzung im Vordergrund. Durch ein entsprechendes Design, die geschickte Wahl der Werkstoffe, durch Miniaturisierung und einen modularen Aufbau sollen der spezifische Ressourcenverbrauch und die spezifische Umweltbelastung minimiert werden. Vernachlässigt werden die ungeheuren Effizienzsteigerungspotentiale, die in der ökonomischen Umsetzung liegen.

\section{Analyse der Verfügungsrechte}

Ein völlig vernachlässigter Faktor in der unternehmerischen Produktgestaltung ist die systematische Analyse der damit verbundenen Verfügungsrechte. Zu oft wird angenommen, daß der Kunde in erster Linie das Eigentum an einem bestimmten materiellen Gut erwerben will. Tatsächlich sind mit dem Eigentum jedoch zahlreiche Verfügungsrechte verbunden, die nicht alle im selben Maße zum Nutzen des Kunden beitragen. Beispiele hierfür sind leicht $\mathrm{zu}$ finden; weder Kundennutzenanalysen noch aufwendige Befragungen müssen dafür durchgeführt werden. Ein Blick in die Mülltonne oder auf den Speicher der Konsumenten genügt: Stapeln sich hier ausgediente Altprodukte, kann man davon ausgehen, daß das Veräußerungsrecht häufig als unangenehme Pflicht zur Entsorgung empfunden wird (siehe Beispiel 1).

\section{Beispiel 1: Rücknahmegarantie}

Eine Rücknahmegarantie für ihre Bürostühle gibt die oberpfälzische Grammer AG. Die Kosten für Rücknahme und das Recycling sind schon heute im Verkaufspreis enthalten. Die Garantie gilt für alle Produktreihen die seit 1990 in den Markt eingeführt wurden und schlögt sich bereits in der Entwicklung never Produkte nieder, die recyclingfreundlich gestaltet werden. Obwohl es bei Grammer ouch Überlegungen zu nutzungsorientierten Dienstleistungen (im Sinne eines "Rent-A-Chair") gibt, steht der Absatz der bestehenden Produkte im Vordergrund, auch wenn das Unternehmen Langlebigkeit und Funktionsorientierung als Ziele benennt.

Das Recht, andere von der Nutzung auszuschließen, ist ein weiteres Merkmal des Eigentums. Pendler, die täglich allein mit ihrem PKW zur Arbeit fahren, machen offensichtlich von diesem Recht Gebrauch. Der Erfolg von Car-Sharing-Initiativen (siehe Beispiel 2) aber zeigt, daß 
eine (am Markt bisher nicht artikulierte) Bereitschaft besteht, dieses Recht mit bestimmten Personengruppen (Nachbarn, Kollegen) zu teilen.

Denken wir diesen Ansatz weiter, so führt die Neuverteilung von Verfügungsrechten $\mathrm{zu}$ einer Aufweichung des Strebens nach Eigentum. Ziel marktlicher Aktivitäten ist dann nicht mehr die Übertragung des Eigentums an einer Sache, sondern die öko-effiziente Stiftung von Kundennut-

\section{Beispiel 2: Car-Pools für Mitarbeiter}

Für die Lufthansa AG galt es wegen akuten Parkplatzmangels, die Parkraumbewirtschaftung neuzugestalten. Do der Neubau eines weiteren Parkhauses zu erheblichen ökonomischen und ökologischen Belastungen geführt hätte, entschied sich die Lufthansa, ihren Mitarbeitern die Nutzung eines speziellen Cor-Pools onzubieten. Heute neh-

men etwa 13.000 Bedienstete der Lufthansa den Frankfurter Cor-Pool mit 430 Fahrzeugen in Anspruch. Dabei zeigt sich, daß eine steigende Zahl von Nutzern nur zu relativ wenig mehr Bedarf an Autos führte. Ungefähr 60 Prozent der Nutzer verwenden das System häufig.

zen durch eine zweckmäßige Übertragung einzelner Verfügungsrechte. Das Nutzungsrecht an einem Sachgut etwa setzt sich oft aus einer Vielzahl von Haupt- und Nebenleistungen zusammen. Diese jeweils separat oder gebündelt anzubieten, kann sowohl für den Kunden als auch für den Anbieter von Vorteil sein. In vielen Fällen ist es auch sinnvoll, wenn Konsumenten den Anbieter am Gewinnaneignungsrecht beteiligen. So wird ein Bauunternehmer viel eher auf Energieeffizienz eines Bauobjektes achten, wenn er während der Nutzungsphase unter Zugrundelegung eines standardisierten Mittelwertes an den Energieeinsparungen beteiligt wird.

\section{- Öko-effziente Produkt- gestaltung als Ergebnis}

Ziel ist es, die Verfügungsrechte an einem Produkt so zu verteilen, daß Kundennutzen und Umweltverträglichkeit maximiert werden. Das Ergebnis ist eine öko-effziente Produktgestaltung. Dafür ist aber eine grundlegende Neuorientierung der Produktpolitik gefordert: Die Kundenbedürfnisse sind zum Ausgangspunkt für die Wahl der technischen und verfügungsrechtlichen Produktgestaltung zu machen. Der Anteil materieller und immaterieller Komponenten am „Leistungsbündel Produkt“ ergibt sich dann als Folge. Je mehr die Verfügungsrechte zwischen
Kunden und Anbieter neu verteilt werden, desto stärker treten die immateriellen Komponenten in den Vordergrund.

Zum anderen ist es notwendig, die heute bestehende Einbahnstraßen-Marktforschung zu einer interaktiven Zwei-Wege-Kommunikation auszubauen. Damit kann es gelingen, Fehlinterpretationen der Bedürfnisstruktur des Kunden zu vermeiden und bislang nicht artikulierte Bedürfnisse zu erkennen. In „Focus Groups“ könnten Unternehmensvertreter mit den Konsumenten diskutieren, welche Leistungen ihren Bedürfnissen am ehesten entsprechen. Dies führt zu einem permanenten Kundendialog und steigert den Grad der Interaktion zwischen dem Konsumenten und dem Anbieter.

Denkt man diesen Weg konsequent zu Ende, wird jeder Leistungsaustausch von intensiver Interaktion begleitet sein. Bei jeder Inanspruchnahme einer Leistung müssen sich Kunde und Anbieter über den Inhalt dieser Leistung erneut austauschen. Dabei wird nicht nur die effiziente Mittelwahl diskutiert, sondern es kommt auch zu einer Verhandlung über den Zweck der Leistung. Ziel der Interaktion ist es, die konkreten Bedürfnisse des Konsumenten bei jeder Nutzung individuell zu berücksichtigen. Weiterhin erlaubt eine starke Interaktion dem Anbieter bereits bei der Entwicklung und Erstellung der Leistung den Kunden einzubinden.

\section{Frage der ökologischen Vorteile}

Nun stellt sich natürlich die Frage, welche ökologischen Vorteile diese Interaktion bringt. Zum einen vermeidet sie, daß Unternehmen Produkte anbieten, die nur teilweise die Bedürfnisse des Kunden befriedigen. Darüber hinaus erlaubt die Interaktion auch, Konsum selbst und die ihm zugrundeliegenden Prämissen zu berücksichtigen: Wer etwa Heizöl konsumiert, hat ja in Wirklichkeit ein Bedürfnis nach Wärme. Möglicherweise ließe sich auch manche Nachfrage nach Transport lösen, wenn das zugrundeliegende Bedürfnis nach „Zugang“ anders befriedigt würde. Somit eröffnet die Interaktion einen erheblichen Spielraum, um Ressourcenverbrauch und ökologische Schäden zu reduzieren. Betrachtet man die beiden Variablen der Leistungsgestaltung - die Verteilung von Verfügungsrechten und die Interaktion - liegt es nahe, eine Matrix zu bilden, um Alternativen aufzuzeigen.

Je nach Ausprägung der Dimensionen sind drei Typen öko-effizienter Dienstleistungen zu unterscheiden: produkt-, nutzungs- und bedürfnisori- entierte. Produktorientierte Leistungen werden zusätzlich zu einem verkauften Produkt angeboten. Hierbei bleiben die meisten Verfügungsrechte beim Nutzer. Die Interaktion ist gering. Ein typisches Beispiel dafür wäre die Rücknahmeund Recyclinggarantie der Grammer AG. Denkbar wären auch ein Reparaturservice oder eine reine Anwendungsberatung.

Nutzungsorientierte Leistungen erhöhen durch Miete oder Leasing bzw. durch gemeinsame Nutzung (Sharing oder Pooling) die Nutzungs-effizienz des Produktes. So hat sich die Lebensdauer und Wartungsfreundlichkeit von Kopiergeräten deutlich erhöht, seit diese fast nur noch in Verbindung mit Wartungsverträgen vermietet werden. Die ökologischen Vorteile der nutzungsorientierten Leistungen liegen z.B. in der erhöhten Lebensdauer, im reduzierten Verbrauch von Energie und Betriebsstoffen oder der Entsorgungsfreundlichkeit, da die Sachleistung am Nutzungsende vom Hersteller zurückgenommen wird.

Bei bedürfnisorientierten Leistungen wird nicht mehr das materielle Gut verkauft sondern das Ergebnis der Leistung, die reine Bedürfnisbefriedigung also. So wird etwa Raumwärme statt Heizöl gehandelt,oder es wird die Dienstleistung Dokumentenvervielfältigung statt eines Fotokopiergerätes verkauft. Hier überläßt der Kunde den größten Teil der Verfügungsrechte dem Anbieter, der ihm nur eine Leistungsgarantie gibt. Durch die hohe Interaktion können die Anforderungen individuell angepaßt werden. Die drei Typen von öko-effizienten Dienstleistungen geben einen groben Überblick über die Gestaltungsmöglichkeiten. Durch eine flexible Zuweisung der Verfügungsrechte und eine variable Gestaltung des Interaktionsgrades lassen sich Produktinnovationen entwickeln, die Kundennutzen und Umweltverträglichkeit öko-effizient verbinden und Unternehmen neue Marktchancen entwickeln.

\section{Anmerkung}

Die beiden Beispiele sowie weitere Fallstudien können in einer ausführlichen Fassung (jeweils 8-10 Seiten) gegen einen Verrechnungsscheck über je 12 Mark bei der Bayreuther Initiative angefordert werden.

Die Autoren
Kai Hockerts und Michael G. Moeller sind Mitglieder der
Bayreuther Initiative für Wirtschaftsökologie e.V.
Kontakt: Bayreuther Initiative für Wirtschaftsökologie
e.V., Universitüt Bayreuth, RW-Fakultät, Postfach
110308, 95422 Bayreuth, Tel./Fax (0921) 55529,
eMail: Bayreuther Initiative@ uni-bayreuth.de


(c) 20I0 Authors; licensee IÖW and oekom verlag. This is an article distributed under the terms of the Creative Commons Attribution Non-Commercial No Derivates License (http://creativecommons.org/licenses/by-nc-nd/3.o/), which permits unrestricted use, distribution, and reproduction in any medium, provided the original work is properly cited. 